\title{
BIG TORELLI GROUPS: GENERATION AND COMMENSURATION
}

\author{
J. ARAMAYONA, T. GHASWALA, A. E. KENT, A. MCLEAY, J. TAO, \\ AND R. R. WINARSKI
}

\begin{abstract}
For any surface $\Sigma$ of infinite topological type, we study the Torelli subgroup $\mathcal{I}(\Sigma)$ of the mapping class group $\operatorname{MCG}(\Sigma)$, whose elements are those mapping classes that act trivially on the homology of $\Sigma$. Our first result asserts that $\mathcal{I}(\Sigma)$ is topologically generated by the subgroup of $\operatorname{MCG}(\Sigma)$ consisting of those elements in the Torelli group which have compact support. In particular, using results of Birman [4], Powell [24], and Putman [25] we deduce that $\mathcal{I}(\Sigma)$ is topologically generated by separating twists and bounding pair maps. Next, we prove the abstract commensurator group of $\mathcal{I}(\Sigma)$ coincides with $\operatorname{MCG}(\Sigma)$. This extends the results for finite-type surfaces $[9,6,7,16]$ to the setting of infinite-type surfaces.
\end{abstract}

\section{INTRODUCTION}

Let $\Sigma$ be a connected oriented surface of infinite topological type - that is, a surface with fundamental group that is not finitely generated. We will also assume the boundary components of $\Sigma$ are compact. The mapping class group of $\Sigma$ is the group:

$$
\operatorname{MCG}(\Sigma)=\operatorname{Homeo}(\Sigma, \partial \Sigma) / \operatorname{Homeo}_{0}(\Sigma, \partial \Sigma),
$$

where $\operatorname{Homeo}(\Sigma, \partial \Sigma)$ is the group of self-homeomorphisms of $\Sigma$ which fix $\partial \Sigma$ pointwise, equipped with the compact-open topology, and $\mathrm{Homeo}_{0}(\Sigma, \partial \Sigma)$ is the connected component of the identity in $\operatorname{Homeo}(\Sigma, \partial \Sigma)$. We equip $\operatorname{MCG}(\Sigma)$ with the quotient topology.

There is a natural homomorphism $\operatorname{MCG}(\Sigma) \rightarrow \operatorname{Aut}\left(H_{1}(\Sigma, \mathbb{Z})\right)$, whose kernel is commonly referred to as the Torelli group $\mathcal{I}(\Sigma)<\operatorname{MCG}(\Sigma)$. While Torelli groups of finite-type surfaces have been the object of intense study (see for example [3, 4, 12, 15, 17, 20, 22, 24, 26]) not much is known about them in the case of surfaces of infinite type. The present article aims to be a first step in this direction.

Generation. In a recent article, Patel-Vlamis [23] give a topological generating set for the pure mapping class group $\operatorname{PMCG}(\Sigma)$ (i.e. the subgroup of $\operatorname{MCG}(\Sigma)$ consisting of those mapping classes which fix every end of $\Sigma$; see Section 2). More concretely, they show that $\operatorname{PMCG}(\Sigma)$ is topologically generated by the subgroup of elements with compact support if $\Sigma$ has at most one end accumulated by genus; otherwise, $\operatorname{PMCG}(\Sigma)$ is topologically 
generated by the union of the set of compactly-supported elements and the set of handle shifts; see Section 2.

Observe that $\mathcal{I}(\Sigma)<\operatorname{PMCG}(\Sigma)$. Denote by $\mathcal{I}_{c}(\Sigma)$ the subgroup of $\mathcal{I}(\Sigma)$ consisting of those elements with compact support, and let $\overline{\mathcal{I}_{c}(\Sigma)}$ be the closure of $\mathcal{I}_{c}(\Sigma)$ in PMCG $(\Sigma)$. Our first result asserts that, for any infinitetype surface $\Sigma$, the set of compactly-supported mapping classes contained in the Torelli group topologically generates the Torelli group:

Theorem 1. For any connected oriented surface $\Sigma$ of infinite type, we have $\mathcal{I}(\Sigma)=\overline{\mathcal{I}_{c}(\Sigma)}$.

Birman [4] and Powell [24] showed that the Torelli group of a closed finite-type surface is generated by separating twists (i.e. Dehn twists about separating curves), plus bounding pair maps (that is, products of twists of the form $T_{\gamma} T_{\delta}^{-1}$, where $\gamma$ and $\delta$ are non-separating but their union separates). Putman then proved that the same is true for finite-type surfaces with boundary [25]. In light of this, an immediate consequence of Theorem 1 is:

Corollary 2. Let $\Sigma$ be a connected oriented surface of infinite topological type. Then $\mathcal{I}(\Sigma)$ is topologically generated by separating twists and boundingpair maps.

Theorem 1 implies $\mathcal{I}(\Sigma)$ is a closed subgroup of $\operatorname{MCG}(\Sigma)$. Since $\operatorname{MCG}(\Sigma)$ is a Polish group [1] and closed subgroups of Polish groups are Polish, we have the following corollary.

Corollary 3. Let $\Sigma$ be a connected oriented surface of infinite topological type. Then $\mathcal{I}(\Sigma)$ is a Polish group.

Commensurations. Recall that, given a group $G$, its abstract commensurator $\operatorname{Comm}(G)$ is the group of equivalence classes of isomorphisms between finite-index subgroups of $G$; here, two isomorphisms are equivalent if they agree on a finite-index subgroup. Observe that there is a natural homomorphism

$$
\operatorname{Aut}(G) \rightarrow \operatorname{Comm}(G)
$$

We will prove:

Theorem 4. For any connected oriented surface $\Sigma$ of infinite topological type and without boundary we have

$$
\operatorname{Comm} \mathcal{I}(\Sigma) \cong \operatorname{Aut} \mathcal{I}(\Sigma) \cong \operatorname{MCG}(\Sigma) .
$$

Before continuing we stress that to the best of our knowledge, it is not known whether the Torelli group of an infinite-type surface has any finiteindex subgroups, and so in particular it is possible that $\operatorname{Comm} \mathcal{I}(\Sigma)$ may in fact coincide a priori with Aut $\mathcal{I}(\Sigma)$. 
Historical context and idea of proof. Theorem 4 was previously known to hold for finite-type surfaces. Indeed, Farb-Ivanov [9] proved it for closed surfaces of genus at least five, which was then extended (and generalized to the Johnson Kernel) by Brendle-Margalit to all closed surfaces of genus at least three $[6,7]$. Kida extended the result of Brendle-Margalit to all finitetype surfaces of genus at least four [16]. Finally, recent work of BrendleMargalit and McLeay has further generalized the result to apply to a large class of normal subgroups of finite-type surfaces [5, 21].

In order to prove the theorem, we closely follow Brendle-Margalit's strategy. First, we adapt ideas of Bavard-Dowdall-Rafi [2] to show that every commensuration of the Torelli group respects the property of being a separating twist or a bounding pair map. From this we deduce that every commensuration induces an automorphism of a combinatorial object called the Torelli complex. This complex was originally introduced, for closed surfaces, by Brendle-Margalit [6], who proved that its automorphism group coincides with the mapping class group; this was later extended by Kida [16] to finite-type surfaces with punctures. Using this, plus an inductive argument due to Ivanov [14], we will show that every automorphism of the Torelli complex of an infinite-type surface is induced by a surface homeomorphism. At this point, Theorem 4 will follow easily using a well-known argument of Ivanov [14].

Acknowledgments. This note is the result of a conversation during the conference "Geometry of Teichmüller spaces and mapping class groups" at the University of Warwick in the Spring of 2018. We are grateful to the organizers, Tara Brendle, Mladen Bestvina, and Chris Leininger for the opportunity to participate. We also thank Dan Margalit for several helpful comments.

We are very thankful to the referee for comments and suggestions that helped improve the paper, especially for an alternate argument establishing one of the containments in Theorem 1; see the end of Section 3. We also thank the referee for telling us about Lemma 5, whose proof is due to Federica Fanoni.

Kent, Tao, and Winarski were partially supported by NSF grants DMS1107452, DMS-1107263, DMS-1107367 RNMS: Geometric Structures and Representation Varieties (the GEAR Network). Aramayona was partially supported by grants RYC-2013-13008 and MTM-2015-67781. Kent acknowledges support from NSF grant DMS-1350075 and the Vilas Trustees at the University of Wisconsin. Tao acknowledges partial support from NSF grant DMS-1440140. Winarski acknowledges partial support from the AMSSimons Travel Grants which are administered by the American Mathematical Society, with support from the Simons Foundation. 


\section{Definitions}

In this section we introduce the main objects needed for the proofs of our results.

2.1. Surfaces. Throughout, by a surface we mean a connected, oriented, second-countable topological surface with (possibly empty) compact boundary. We say that $\Sigma$ has finite type if its fundamental group is finitely generated; otherwise, we say that $\Sigma$ has infinite type. In the finite type case, we will sometimes use the notation $\Sigma=\Sigma_{g, p}^{b}$, where $g, p$, and $b$ are, respectively, the genus, the number of punctures, and the number of boundary components of $\Sigma$. In this case, we define the complexity of $\Sigma$ to be the integer $\xi(\Sigma)=3 g-3+p+b$.

The space of ends of $\Sigma$ is the set

$$
\operatorname{Ends}(\Sigma)=\lim _{\longleftarrow} \pi_{0}(\Sigma \backslash K),
$$

where the inverse limit is taken over the set of compact subsets $K \subset \Sigma$, directed with respect to inclusion. Here, the topology on $\operatorname{Ends}(\Sigma)$ is given by the limit topology obtained by equipping each $\pi_{0}(\Sigma \backslash K)$ with the discrete topology. See [27] for further details.

We say that $e$ in $\operatorname{Ends}(\Sigma)$ is accumulated by genus if every neighborhood of $e$ has infinite genus; otherwise, we say that $e$ is planar. In particular, observe that every puncture of $\Sigma$ is a planar end. We denote by $\operatorname{Ends}_{g}(\Sigma)$ the subset of Ends $(\Sigma)$ consisting of ends accumulated by genus. It is a classical theorem (see [27] for a discussion and proof) that the homeomorphism type of $\Sigma$ is determined by the tuple

$$
\left(g(\Sigma), b(\Sigma), \operatorname{Ends}(\Sigma), \operatorname{Ends}_{g}(\Sigma)\right)
$$

where $g(\Sigma)$ and $b(\Sigma)$ denote the genus and the number of boundary components of $\Sigma$.

2.2. Curves and domains. By a curve on $\Sigma$ we mean the free homotopy class of a simple closed curve that does not bound a disk or a disk containing a single planar end of $\Sigma$. Abusing notation, we will not make any distinction between a curve and any of its representatives.

We say that a curve $\gamma$ is separating if $\Sigma \backslash \gamma$ has two connected components; otherwise, we say that $\gamma$ is non-separating. We say that two curves are disjoint if they have disjoint representatives in $\Sigma$. A multicurve is a set of pairwise disjoint curves. Given two curves $\alpha$ and $\beta$, we denote by i $(\alpha, \beta)$ their geometric intersection number. The intersection number between two multicurves is defined additively.

A domain $Y$ in $\Sigma$ is a closed subset which is itself a surface and the inclusion map is a proper, $\pi_{1}$-injective embedding. Note that domains are only defined up to isotopy. A subsurface of $\Sigma$ is a disjoint union of domains. The following definition appears in [2]. 
Definition. A domain $Y$ of $\Sigma$ is called principal if $Y$ has finite-type and every component of $\Sigma \backslash Y$ has infinite-type.

The following lemma was communicated to us by the referee. The statement and the proof are due to Federica Fanoni.

Lemma 5. Let $\sigma$ be multicurve. Then there are curves realizing the homotopy classes in $\sigma$ which do not accumulate in any compact set of $\Sigma$ if and only if for every $\alpha \notin \sigma$, the set $\{\beta \in \sigma \mid \mathrm{i}(\alpha, \beta) \neq 0\}$ is finite.

Proof. The forward direction is clear so we will focus on the other direction. Choose a complete hyperbolic metric on $\Sigma$ without half planes and realize all curves as geodesics. The assumption on the metric implies that we can decompose $\Sigma$ into pairs of pants with geodesic boundary, funnels and/or cusps (as shown in [13]). If the geodesic representative of $\sigma$ has an accumulation point, then we can find a pair of pants $P$ of $\Sigma$ containing such a point. However, by assumption, there are only finitely many curves of $\sigma$ that intersect the boundary of $P$. Thus, only finitely many curves of $\sigma$ intersect $P$. In particular, they cannot accumulate in $P$, a contradiction.

Given an element $f \in \operatorname{MCG}(\Sigma)$, there is a canonical (possibly empty) multicurve $\partial f$ in $\Sigma$ for which $f(\partial f)=\partial f$, defined as follows. Let $\mathcal{O}(f)$ be the set of curves $\alpha$ such that $\left\{f^{k}(\alpha) \mid k \in \mathbb{Z}\right\}$ is finite. Then $\partial f$ is the set of curves in $\mathcal{O}(f)$ that are disjoint from all other elements of $\mathcal{O}(f)$. See [11][§2] and also [2] for further details.

Given $f \in \operatorname{MCG}(\Sigma)$ and a subsurface $Y$, we say $Y$ supports $f$ or $f$ is supported on $Y$ if $f$ can be realized by a homeomorphism which is the identity outside of $Y$. An element $f \in \operatorname{MCG}(\Sigma)$ is said to have compact support if there is a compact subsurface that supports $f$. Similarly, $f$ has finite support if there is a subsurface of finite-type that supports $f$. If $f$ is a product of Dehn twists about a multicurve $\alpha$, then we will also say $\alpha$ supports $f$.

The following statement follows from [2]. The first assertion is Lemma 2.6 , while the second follows from its proof.

Lemma 6. If $f \in \operatorname{MCG}(\Sigma)$ is nontrivial and has finite support, then $f$ has infinite order and $\partial f$ is a nonempty multicurve in $\Sigma$. Furthermore, let $Y$ be the (finite) union of all finite-type domains in $\Sigma \backslash \partial f$, then $Y$ supports $f$.

2.3. Pure mapping classes. The pure mapping class group $\operatorname{PMCG}(\Sigma)$ is the normal subgroup of $\operatorname{MCG}(\Sigma)$ whose elements fix every end of $\Sigma$.

The set of compactly-supported mapping classes $\mathrm{PMCG}_{c}(\Sigma)$ forms a subgroup of $\operatorname{PMCG}(\Sigma)$ which is normal in $\operatorname{MCG}(\Sigma)$. Note that if $f$ lies in $\operatorname{PMCG}(\Sigma)$ and $f$ has finite support, then it necessarily has compact support. Since $\mathrm{PMCG}_{c}(\Sigma)$ is a direct limit of pure mapping class groups of compact surfaces, a classical result due to Dehn and Lickorish (see [10, Section 4], for instance) implies that $\mathrm{PMCG}_{c}(\Sigma)$ is generated by Dehn twists. 
2.4. Handle Shifts. For any subgroup $\Gamma<\operatorname{MCG}(\Sigma)$, we denote by $\bar{\Gamma}$ its topological closure in $\mathrm{MCG}(\Sigma)$.

Patel-Vlamis introduced handle shifts and showed that handle shifts and Dehn twists topologically generate $\operatorname{PMCG}(\Sigma)$ [23]. Subsequently, in [1] it was shown that $\mathrm{PMCG}(\Sigma)=\overline{\mathrm{PMCG}_{c}(\Sigma)} \rtimes H$, where $H$ is a particular subgroup isomorphic to a direct product of pairwise commuting handle shifts. We now recall the definition of a handle shift.

Let $\Lambda$ be the surface obtained from $\mathbb{R} \times[-1,1]$ by removing disks of radius $\frac{1}{4}$ centered at $(t, 0)$ for $t$ in $\mathbb{Z}$ and gluing in a torus with one boundary component, identifying the boundary of the torus with the boundary of the removed disk. Let $\sigma: \Lambda \rightarrow \Lambda$ be the homeomorphism that shifts the handle at $(t, 0)$ to the handle at $(t+1,0)$, and is the identity on $\mathbb{R} \times\{-1,1\}$ (see [1] or [23] for an image of such a homeomorphism). The isotopy class of $\sigma$ is called a handle shift of $\Lambda$.

An element $h$ in $\operatorname{MCG}(\Sigma)$ is a handle shift if there exists a proper embedding $\iota: \Lambda \rightarrow \Sigma$ which induces an injective map on ends, and such that $[h]=[\delta]$ where $\left.\delta\right|_{\iota(\Lambda)}=\sigma$ and $\delta$ is the identity outside $\iota(\Lambda)$. As a consequence of our definition, we must have $\left|\operatorname{Ends}_{g}(\Sigma)\right| \geq 2$; also, for each handle shift there is an attracting end $\epsilon_{+}$and a repelling end $\epsilon_{-}$in $\operatorname{Ends}_{g}(\Sigma)$, and they are distinct.

We say a handle shift $h$ with attracting end $\epsilon_{+}$and repelling end $\epsilon_{-}$is dual to a separating curve $\gamma$ if each component of $\Sigma \backslash \gamma$ contains exactly one of $\epsilon_{+}$and $\epsilon_{-}$.

2.5. Principal exhaustions. We now introduce a minor modification of the notion of principal exhaustion from $[1,2]$.

Definition. A principal exhaustion of $\Sigma$ is an infinite sequence of principal domains $\left\{P_{1}, P_{2}, \ldots\right\}$ such that, for every $i \geq 1$, one has:

(1) $P_{i} \subset P_{i+1}$,

(2) every component of $\partial P_{i}$ is separating

(3) no component of $\partial P_{i}$ is isotopic to a component of $\partial P_{i+1}$, and

(4) $\Sigma=\bigcup P_{i}$.

Lemma 7. Let $\Sigma$ be a connected infinite-type surface and let $\left\{P_{i}\right\}$ be a principal exhaustion of $\Sigma$. Then for all $i$, we have:

- for all $j>i, H_{1}\left(P_{j}\right) \cong H_{1}\left(P_{i}\right) \oplus M$ for some $M<H_{1}\left(P_{j} \backslash P_{i}\right)$

- $H_{1}(\Sigma) \cong H_{1}\left(P_{i}\right) \oplus M^{\prime}$ for some $M^{\prime}<H_{1}\left(\Sigma \backslash P_{i}\right)$

Proof of lemma. We will let $W$ be either $P_{j}$ or $\Sigma$ to prove both cases simultaneously.

Let $\partial_{1} P_{i}, \ldots \partial_{m} P_{i}$ be the boundary components of $P_{i}$.

Since every component of $\Sigma-P_{i}$ is of infinite type, every component of $\overline{W-P_{i}}$ either contains an end of $\Sigma$ or a boundary component of $W$. So there is a collection of pairwise disjoint rays and arcs $\gamma_{1}, \ldots, \gamma_{m}$ properly embedded in $\overline{W-P_{i}}$ such that $\gamma_{k} \cap \partial_{k} P_{i}$ is a single point for all $k$. 

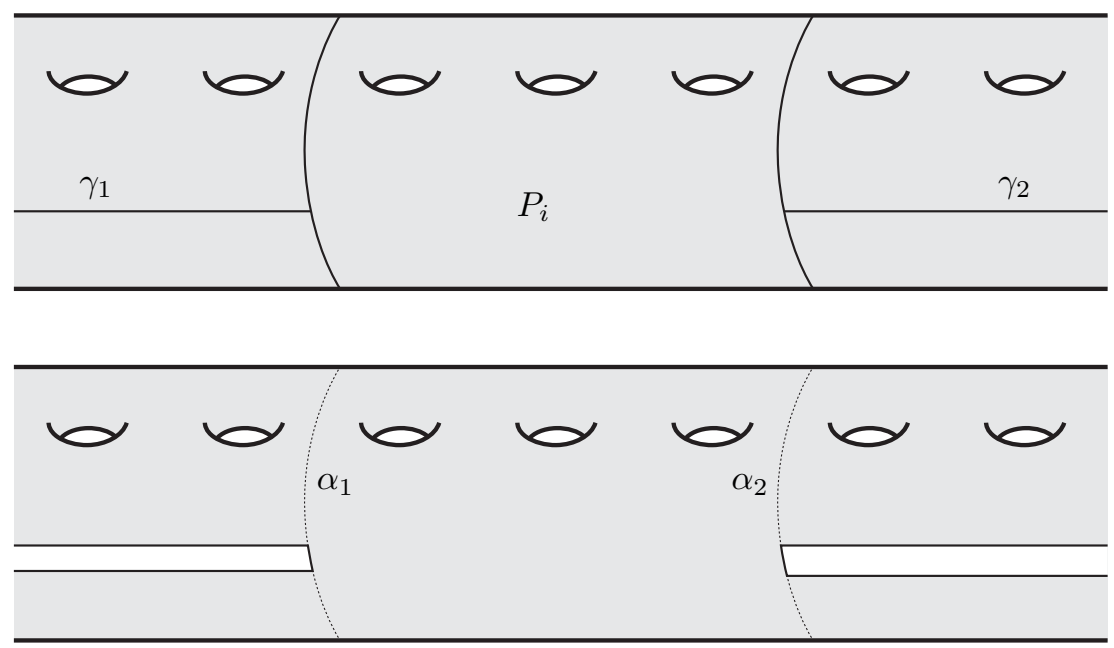

Figure 1. Pictured at the top is the surface $W$, the subsurface $P_{i}$, and the $\operatorname{arcs} \gamma_{1}, \ldots, \gamma_{m}$. Below is the surface $\Delta$, obtained by deleting open neighborhoods of the interiors of the $\gamma_{k}$. The $\alpha_{k}$ are the dotted arcs.

By the Regular Neighborhood Theorem, we may deformation retract $W$ along the $\gamma_{k}$, fixing $P_{i}$ throughout, to obtain a subsurface $\Delta$ homotopy equivalent to $W$ that contains $P_{i}$ and such that $P_{i} \cap \overline{\Delta-P_{i}}$ is a disjoint union of $\operatorname{arcs} \alpha_{1}, \ldots, \alpha_{m}$, as pictured in Figure 1.

Consideration of the Mayer-Vietoris sequence gives us an exact sequence

$$
0 \rightarrow H_{1}\left(P_{i}\right) \oplus H_{1}\left(\Delta-P_{i}\right) \longrightarrow H_{1}(W) \stackrel{\partial}{\longrightarrow} H_{0}\left(\alpha_{1} \sqcup \cdots \sqcup \alpha_{m}\right) .
$$

This gives us the direct sum decomposition of $H_{1}(W)$. Since $\partial_{\ell} P_{i}$ are separating, then so are the $\alpha_{\ell}$. This implies that the boundary map $\partial$ is zero, and since $H_{1}\left(\Delta-P_{i}\right)$ is naturally a subgroup of $H_{1}\left(W-P_{i}\right)$, the proof is complete.

\section{Compactly generating the Torelli group}

Let $\Sigma$ be an infinite-type surface. We define the compactly supported Torelli group

$$
\mathcal{I}_{c}(\Sigma):=\{f \in \mathcal{I}(\Sigma) \mid f \text { has compact support }\} .
$$

The aim of this section is to prove the first main result of the introduction, whose statement we now recall:

Theorem 1. For any connected oriented surface $\Sigma$ of infinite type, we have $\mathcal{I}(\Sigma)=\overline{\mathcal{I}_{c}(\Sigma)}$.

Remark. The referee suggested an alternate way of proving that every element of $\mathcal{I}(\Sigma)$ is a limit of mapping classes with compact support; we give this argument at the end of this section. However, we have decided to keep 
our original argument, phrased in terms of a new type of mapping class (pseudo handle shifts, see below), which may be of independent interest.

We will need to know that certain, possibly infinite, products of handle shifts are inaccessible by compactly supported mapping classes. For a general product of handle shifts, this is too much to hope for. For example, in a surface with two ends, the product of two commuting handle shifts with opposite dynamics is a limit of compactly supported classes.

More generally, there are products of infinitely many commuting handle shift that are limits of compactly supported classes. For example, there is the "boundary leaf shift," which we now explain.

Example (Boundary leaf shift). Start with an infinite regular tree $\mathcal{T}$ properly embedded in the hyperbolic plane $\mathbb{H}^{2}$ with boundary a Cantor set in $\partial \mathbb{H}^{2}$. Orient $\partial \mathbb{H}^{2}$ counterclockwise. Build a surface by taking the boundary of a regular neighborhood of $\mathcal{T}$ in $\mathbb{H}^{2} \times \mathbb{R}$ and attach handles periodically (in the hyperbolic metric) along each side of $\mathcal{T}$, see Figure 2. The orientation on $\partial \mathbb{H}^{2}$ defines a product $\mathcal{H}$ of handle shifts by shifting the handles in each region of $\mathbb{H}^{2}-\mathcal{T}$ in the clockwise direction.

To see the boundary leaf shift is in $\overline{\mathrm{PMCG}_{c}(\Sigma)}$, pick a basepoint $*$ in $\mathcal{T}$ and consider the $n$-neighborhood $B(n)$ of $*$ in $\mathcal{T}$. Then we may move the handles incident to $B(n)$ around in a counterclockwise fashion to get a compactly supported class $f_{n}$ in $\mathrm{PMCG}_{c}(\Sigma)$. The sequence $\left\{f_{n}\right\}$ converges to the boundary leaf shift.

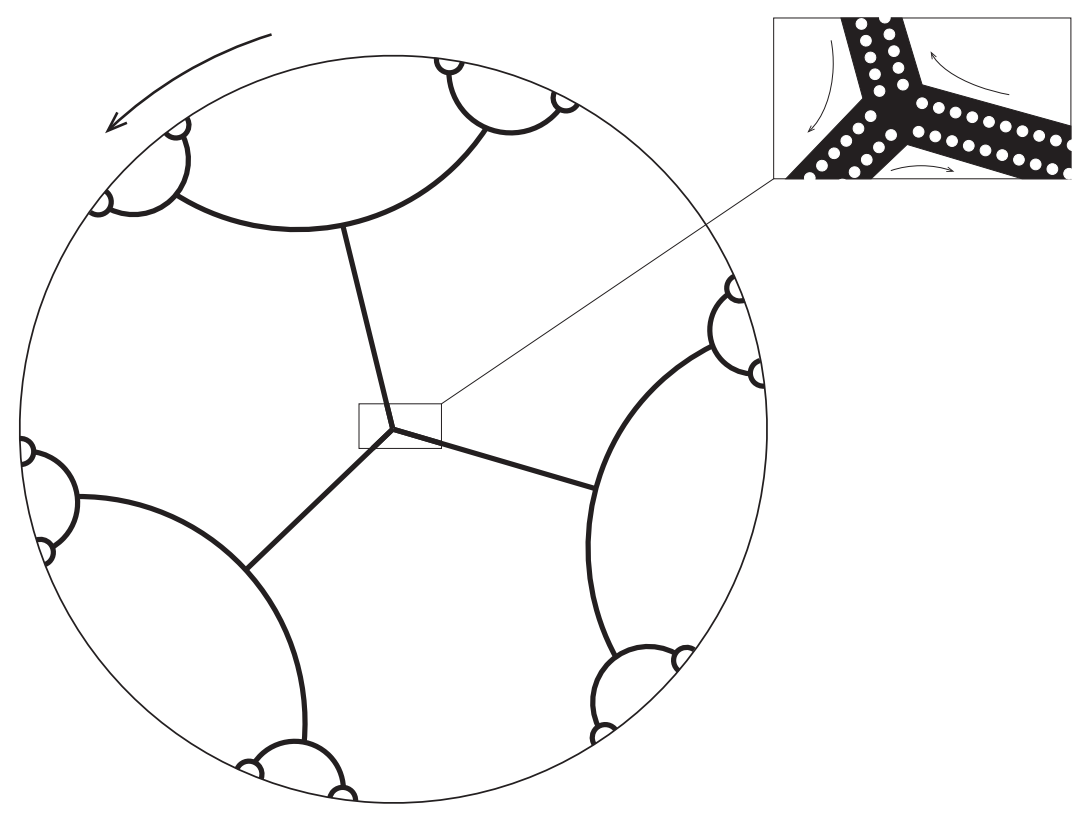

Figure 2. The boundary leaf shift. 
Let $\gamma$ be a separating curve in $\Sigma$ whose complementary components are both noncompact. Let $\Sigma_{-}$and $\Sigma_{+}$be the closures of the two components of $\Sigma-\gamma$. By the same argument as in Lemma $7, \Sigma$ deformation retracts to a subspace homeomorphic to $X \vee \gamma \vee Y$, where $X$ and $Y$ are subspaces of $\Sigma_{-}$and $\Sigma_{+}$, respectively. It follows that $H_{1}(\Sigma)$ splits as $A \oplus\langle\gamma\rangle \oplus B$, where $A=H_{1}(X)$ and $B=H_{1}(Y)$.

Similarly, if $h$ is a handle shift dual to $\gamma$, then $H_{1}(\Sigma) \cong L \oplus\langle\gamma\rangle \oplus$ $H_{1}(\operatorname{supp}(h)) \oplus R$, where $L$ and $R$ are subgroups of $A$ and $B$.

Definition (Pseudo handle shift). We say that a mapping class $\mathcal{H}$ is a pseudo handle shift dual to a separating curve $\gamma$ with associated handle shift $h$ if the following hold:

(1) $h$ is a handle shift dual to $\gamma$

(2) $\mathcal{H}_{*}$ agrees with $h_{*}$ on $H_{1}(\operatorname{supp}(h))$

(3) $\mathcal{H}_{*}([\gamma])=[\gamma]$

(4) $\mathcal{H}_{*}(L)<A$

(5) $\mathcal{H}_{*}(R)<B$

In what follows, we always assume that the repelling end of $h$ is on the " $A$-side."

Examples. Let $h$ be a handle shift dual to a separating curve $\gamma$. Then $h$ is itself a pseudo handle shift dual to $\gamma$ (with associated handle shift $h$ ).

For a less trivial example, take a connected component $X$ of $\Sigma \backslash(\gamma \cup$ $\operatorname{supp}(h))$. Then the composition $h$ with any element $g \in \operatorname{MCG}(\Sigma)$ supported on $X$ is a pseudo handle shift associated to $h$. In particular, if $h^{\prime}=\prod h_{i}$ is a product of commuting handle shifts $h_{i}$ with dual curves $\gamma_{i}$, such that $h$ and $h^{\prime}$ commute, $\operatorname{supp}\left(h^{\prime}\right)$ is disjoint from $\gamma$ and $\operatorname{supp}(h)$ is disjoint from all $\gamma_{i}$, then the composition $k=h \circ h^{\prime}$ is a pseudo handle shift associated to $h$.

Note that since pseudo handle shifts are defined by their action on homology, if $f_{*}=\mathcal{H}_{*}$ for a pseudo handle shift $\mathcal{H}$ associated to $h$, then $f$ is also a pseudo handle shift associate to $h$.

Theorem 8 (Pseudo handle shifts are unapproachable). A pseudo handle shift $\mathcal{H}$ dual to a separating curve $\gamma$ is not a limit of compactly supported mapping classes.

Proof. Let $h$ be the associated handle shift dual to $\gamma$. Let $\epsilon_{-}$and $\epsilon_{+}$be the ends of $\Sigma$ corresponding to the repelling and attracting ends of $h$, respectively, and let $\Sigma_{-}$and $\Sigma_{+}$be the complementary components of $\Sigma-\gamma$ containing $\epsilon_{-}$and $\epsilon_{+}$, respectively. Choose some principal exhaustion $\left\{P_{i}\right\}$ of $\Sigma$, and let $\Sigma_{-}^{i}=\left(\Sigma-P_{i}\right) \cap \Sigma_{-}$and $\Sigma_{+}^{i}=\left(\Sigma-P_{i}\right) \cap \Sigma_{+}$.

The curve $\gamma$ partitions the space of ends into two closed subspaces $E_{-}$ and $E_{+}$of which $\Sigma_{-}$and $\Sigma_{+}$are neighborhoods, respectively. The subsurfaces $\Sigma_{-}^{i}$ and $\Sigma_{+}^{i}$ are also neighborhoods of $E_{-}$and $E_{+}$. Since $\mathcal{H}$ is pure, $\mathcal{H}\left(\Sigma_{-}^{i}\right)$ and $\mathcal{H}\left(\Sigma_{+}^{i}\right)$ are also neighborhoods of $E_{-}$and $E_{+}$. Since $E_{-}$and $E_{+}$ are disjoint, the intersection of the closures of $\mathcal{H}\left(\Sigma_{ \pm}^{i}\right)$ and $\Sigma_{\mp}$ is compact, 
and since the $\Sigma_{ \pm}^{i}$ are nested and have empty intersection, we may take $i$ large enough so that the term $P_{i}$ in our principal exhaustion contains $\gamma$ and satisfies $\mathcal{H}\left(\Sigma_{ \pm}^{i}\right) \cap \Sigma_{\mp}$ is empty.

The handle shift $h$ is supported on a strip $\mathcal{S}$ with equally spaced handles and standard basis $\left\{\alpha_{p}, \beta_{p}\right\}_{k \in \mathbb{Z}}$ of $H_{1}(\mathcal{S})$ so that $h_{*}\left(\alpha_{p}\right)=\alpha_{p+1}$ and $h_{*}\left(\beta_{p}\right)=$ $\beta_{p+1}$. We choose once and for all curves in $\mathcal{S}$ representing these classes. After reindexing the $\alpha_{p}$ and $\beta_{p}$ by translating $p$, we assume that $\alpha_{1}$ and $\beta_{1}$ lie in $\Sigma_{-}^{i}$. Since $\alpha_{p}$ and $\beta_{p}$ tend to $\epsilon_{+}$, there is some $j>1$ such that $\alpha_{j}$ and $\beta_{j}$ lie in $\Sigma_{+}^{i}$.

Suppose that $\mathcal{H}$ is a limit of compactly supported $\mathcal{H}_{n}$. Pick $n$ large enough so that $\mathcal{H}_{n}$ agrees with $\mathcal{H}$ on $P_{i}$ and so that $\mathcal{H}_{n *}$ agrees with $h_{*}$ on both $H_{1}\left(P_{i}\right)$ and $\left\langle\alpha_{1}, \beta_{1}, \ldots, \alpha_{j}, \beta_{j}\right\rangle$. Let $P_{k}$ be some term in the exhaustion with $k \geq i$ that contains the support of $\mathcal{H}_{n}$.

We have a direct sum decomposition

$$
H_{1}\left(P_{k}\right) \cong \mathbb{Z}^{\ell} \oplus \mathbb{Z}^{2 j} \oplus \mathbb{Z}^{r}
$$

where $\mathbb{Z}^{\ell}$ is a subgroup of $H_{1}\left(\Sigma_{-}\right) \oplus\langle\gamma\rangle, \mathbb{Z}^{2 j}=\left\langle\alpha_{1}, \beta_{1}, \ldots, \alpha_{j}, \beta_{j}\right\rangle$, and $\mathbb{Z}^{r}$ is a subgroup of $H_{1}\left(\Sigma_{+}\right)$. Picking a basis $\left\langle x_{1}, \ldots, x_{\ell}, \alpha_{1}, \beta_{1}, \ldots, \alpha_{j}, \beta_{j}, y_{1}, \ldots, y_{r}\right\rangle$ for $H_{1}\left(P_{k}\right)$ compatible with this decomposition, we see that $\mathcal{H}_{n *}$ has a block decomposition:

$$
\mathcal{H}_{n *}=\underset{\ell}{2 j-2} \underset{r}{2 j}\left(\begin{array}{cccc}
* & 2 j-2 & 2 & r \\
* & \mathbf{0} & \mathbf{0} & Y \\
* & I & \mathbf{0} & Z \\
X & \mathbf{0} & A & B
\end{array}\right)
$$

By properties (4) and (5) of a pseudo handle shift, and our choice of $i$, the blocks $X, Y$, and $Z$ are all zero. So the matrix is:

$$
\mathcal{H}_{n *}=\begin{gathered}
\ell \\
\ell \\
2 j-2 \\
2 \\
r
\end{gathered}\left(\begin{array}{cccc}
* & 2 j-2 & 2 & r \\
* & \mathbf{0} & \mathbf{0} & \mathbf{0} \\
* & I & \mathbf{0} & * \\
\mathbf{0} & \mathbf{0} & A & B
\end{array}\right)
$$

This matrix is column equivalent to:

$$
\mathcal{H}_{n *}=\underset{\ell}{\ell} \begin{array}{cccc}
\ell & 2 j-2 & 2 & r \\
2 j-2 \\
2 \\
r
\end{array}\left(\begin{array}{cccc}
* & \mathbf{0} & \mathbf{0} & \mathbf{0} \\
* & \mathbf{0} & \mathbf{0} & \mathbf{0} \\
* & I & \mathbf{0} & \mathbf{0} \\
\mathbf{0} & \mathbf{0} & A & B
\end{array}\right)
$$

But the matrix $\left[\begin{array}{ll}A & B\end{array}\right]$ is an $r \times(r+2)$ matrix, and so its Jordan form cannot have a pivot in every column. So the matrix for $\mathcal{H}_{n *}$ is equivalent 
to a matrix with a zero column. But $\mathcal{H}_{n *}$ is an isomorphism, and this contradiction completes the proof.

We are now ready to prove Theorem 1 .

Proof of Theorem 1. We will first show that $\mathcal{I}(\Sigma)<\overline{\operatorname{PMCG}_{c}(\Sigma)}$. By $[23$, Theorem 1], we only need to consider the case when $\Sigma$ has at least two ends accumulated by genus. We observe that $\mathcal{I}(\Sigma)<\operatorname{PMCG}(\Sigma)$. Let $g$ be in $\operatorname{PMCG}(\Sigma)$ so that $g$ is not a limit of compactly supported mapping classes. We show that $g$ is not in $\mathcal{I}(\Sigma)$.

By Theorem 3 and Corollary 4 from [1], $g$ can be written $g=f k^{-1}$ where $f$ is a limit of compactly supported classes and $k$ is a product of pairwise commuting handle shifts $h_{i}$. The handle shift $h_{i}$ has the property that the support of $h_{i}$ is disjoint from the dual curve $\gamma_{j}$ for $h_{j}$ whenever $i \neq j$. Thus, for any $i, k$ is a pseudo-handle shift dual to $\gamma_{i}$ associated to $h_{i}$ (see Examples after the definition of pseudo handle shifts). If $g$ were in Torelli, then $f_{*}=g_{*} k_{*}=k_{*}$, which implies $f$ is a pseudo handle shift, but this violates Theorem 8 . This shows

$$
\mathcal{I}(\Sigma)<\overline{\operatorname{PMCG}_{c}(\Sigma)}
$$

If $\phi_{n}$ is a sequence in $\mathcal{I}_{c}(\Sigma)$ that converges to $\phi$, then $\phi$ lies in $\mathcal{I}(\Sigma)$, since $\phi_{n}(\alpha)$ eventually agrees with $\phi(\alpha)$ for any given simple closed curve $\alpha$. So

$$
\overline{\mathcal{I}_{c}(\Sigma)}<\mathcal{I}(\Sigma) .
$$

For the other containment, let $\phi$ be an element of $\mathcal{I}(\Sigma)$ and let $\left\{\psi_{n}\right\}$ be a sequence in $\mathrm{PMCG}_{c}(\Sigma)$ converging to $\phi$. We would like to convert $\psi_{n}$ into a sequence of compactly supported $\phi_{n}$ in $\mathcal{I}(\Sigma)$ converging to $\phi$. The idea is that the homology classes affected by $\psi_{n}$ must move further and further away from a given basepoint, and so we can precompose the $\psi_{n}$ with a mapping class supported far from the basepoint to produce the desired $\phi_{n}$.

Fix a principal exhaustion $\left\{P_{i}\right\}$ of $\Sigma$. For each $i$, pick a $j>i$ such that $P_{j}$ contains $\phi^{-1}\left(P_{i}\right)$. Pick an $N$ large enough so that, for all $n \geq N$, the map $\psi_{n}$ has a representative that agrees with a fixed representative of $\phi$ on $P_{j}$. Note that $\psi_{n *}$ agrees with $\phi_{*}$ on $H_{1}\left(P_{j}\right)$. Pick a $k>j$ such that $P_{k}$ contains the support of $\psi_{n}$.

By Lemma 7, we have $H_{1}\left(P_{k}\right) \cong H_{1}\left(P_{i}\right) \oplus Q \oplus R$ for some $Q$ a subgroup of $H_{1}\left(P_{j}-P_{i}\right)$ and $R$ a subgroup of $H_{1}\left(P_{k}-P_{j}\right)$. Let $\alpha$ be element of $H_{1}\left(P_{k}\right)$ and write $\alpha=\gamma+\mu+\nu$ where $\gamma, \mu$, and $\nu$ are in $H_{1}\left(P_{i}\right), Q$, and $R$, respectively. So $\psi_{n *}(\alpha)=\gamma+\mu+\psi_{n *}(\nu)$.

The class $\nu$ is represented by a $1-$ manifold $\mathcal{N}$ in $P_{k}-P_{j}$. By our choice of $j$ and $n$, the 1-manifold $\psi_{n}(\mathcal{N})$ is disjoint from $P_{i}$. So $\psi_{n *}(\nu)$ is in $Q \oplus R$. Therefore $\psi_{n *}: H_{1}\left(P_{k}\right) \rightarrow H_{1}\left(P_{k}\right)$ may be represented by a square matrix

$$
A=\left[\begin{array}{ll}
I & \mathbf{0} \\
\mathbf{0} & B
\end{array}\right]
$$


where $I$ is the identity on $H_{1}\left(P_{i}\right)$ and $B$ is a square matrix. Since $A$ is the induced map on homology associated to a homeomorphism of $P_{k}$, it is invertible and respects the intersection form, and so the same is true of $B$.

We claim that the matrix $B$ is represented by a homeomorphism $F^{\prime}$ : $\overline{P_{k}-P_{i}} \rightarrow \overline{P_{k}-P_{i}}$ that is the identity on $\partial P_{i} \cap \overline{P_{k}-P_{i}}$. To see this, note that $B$ preserves the intersection form and the homology classes of the boundary components of each component of $\overline{P_{k}-P_{i}}$. Let $X$ be the surface obtained from $\overline{P_{k}-P_{i}}$ by capping off all the boundary components with disks. The homology of $X$ is a quotient of that of $\overline{P_{k}-P_{i}}$, and $B$ induces an automorphism of the homology of $X$ that preserves the intersection form. There is a homeomorphism $X \rightarrow X$ inducing this automorphism that preserves each component of $X$, by Burkhardt's theorem [8]. By an isotopy, we may assume that Burkhardt's homeomorphism of $X$ fixes, point-wise, small disks around the centers of the disks we added to construct $X$. Restricting this to $\overline{P_{k}-P_{i}}$ is the desired $F^{\prime}$.

We extend $F^{\prime}$ by the identity to all of $\Sigma$ and call the result $F$.

Now consider the homeomorphism $\phi_{n}=\psi_{n} \circ F^{-1}$. By the construction of $F$, this homeomorphism acts trivially on the homology of $\Sigma$, and agrees with $\psi_{n}$ on $P_{i}$.

This completes the proof.

We finish this section with the alternate proof of the fact that $\mathcal{I}(\Sigma)<$ $\overline{\mathrm{PMCG}_{c}(\Sigma)}$, as suggested by the referee. Suppose, for contradiction, that there is $f \in \mathcal{I}(\Sigma)$ which may not be expressed as the limit of a sequence of mapping classes with compact support. A consequence of [1, Theorem 4.5] is that there is a separating curve $\gamma \subset \Sigma$ such that $\gamma$ and $f(\gamma)$ have different topological types in every compact subsurface of $\Sigma$ containing them. In particular, they induce different splittings of $H_{1}(\Sigma, \mathbb{Z})$, which contradicts the fact that $f$ acts trivially on homology.

\section{Abstract commensurators of the Torelli group}

In this section we prove Theorem 4. As in the statement of the theorem, throughout this section we will assume that the surface $\Sigma$ has empty boundary. As mentioned in the introduction, the first step of the argument consists of proving that an element of $\operatorname{Comm} \mathcal{I}(\Sigma)$ induces a simplicial automorphism of a combinatorial object associated to $\Sigma$, called the Torelli complex, introduced by Brendle-Margalit in [6].

4.1. Torelli complex. Recall that the curve complex of $\Sigma$ is the (infinitedimensional) simplicial complex whose vertex set is the set of isotopy classes of curves in $\Sigma$, and where a collection of vertices spans a simplex if and only if the corresponding curves are pairwise disjoint. The curve complex was used by Ivanov [14], Korkmaz [18], and Luo [19] to prove that, for all but a few finite-type surfaces $\Sigma$,

$$
\operatorname{Comm} \operatorname{MCG}(\Sigma) \cong \operatorname{Aut} \operatorname{MCG}(\Sigma) \cong \operatorname{MCG}(\Sigma) .
$$


Subsequently, Bavard-Dowdall-Rafi [2] established the analogous result for every infinite-type surface. In a similar fashion, Farb-Ivanov [9], BrendleMargalit [6, 7, 5], and Kida [16] proved that, for all but a few finite-type surfaces,

$$
\operatorname{Comm} \mathcal{I}(\Sigma) \cong \operatorname{Aut} \mathcal{I}(\Sigma) \cong \operatorname{MCG}(\Sigma) .
$$

Here, we will adapt the ideas of Brendle-Margalit [6] to the infinite-type setting. Given an infinite-type surface $\Sigma$, we define its Torelli complex to be the (infinite-dimensional) simplicial complex whose vertex set is the set of isotopy classes of separating curves and bounding pairs in $\Sigma$, and where a collection of vertices spans a simplex if and only if the corresponding curves are pairwise disjoint. In order to relax notation, we will blur the distinction between vertices of $\mathcal{T}(\Sigma)$ and the curves (or multicurves) they represent.

The Torelli complex of a finite-type surface is connected [9]. As a consequence, the same holds for the Torelli complex of an infinite-type surface also. We record the following observation as a separate lemma, as we will need to make use of it later:

Lemma 9. The Torelli complex $\mathcal{T}(\Sigma)$ has infinite diameter if and only if $\Sigma$ has finite type.

Proof. If $\Sigma$ has finite type, a slick limiting argument due to Feng Luo (see the comment after Proposition 4.6 of [20]) shows that the curve complex has infinite diameter. The obvious adaptation of this method to the case of the Torelli complex also implies that $\mathcal{T}(\Sigma)$ has infinite diameter.

For the other direction, suppose $\Sigma$ has infinite type. Since curves are compact, given multicurves $\gamma, \delta \subset \Sigma$, we can find a separating curve $\eta \subset \Sigma$ which is disjoint from both $\gamma$ and $\delta$. In particular, $\mathcal{T}(\Sigma)$ has diameter two.

4.2. Automorphisms of the Torelli complex. Denote by Aut $\mathcal{T}(\Sigma)$ the group of simplicial automorphisms of $\mathcal{T}(\Sigma)$, and observe that there is a natural homomorphism $\operatorname{MCG}(\Sigma) \rightarrow \operatorname{Aut} \mathcal{T}(\Sigma)$. We want to prove:

Theorem 10. Let $\Sigma$ be an infinite-type surface without boundary. The natural homomorphism $\operatorname{MCG}(\Sigma) \rightarrow \operatorname{Aut} \mathcal{T}(\Sigma)$ is an isomorphism.

As noted above, the finite-type case is due to Brendle-Margalit $[6,7,5]$ and Kida [16]. Indeed, the notion of sides which is used in this section is adapted from arguments that may be found in Brendle-Margalit [6], and which find their way back to ideas of Ivanov [14].

Sides. Recall that the link of a vertex $v$ of a simplicial complex $X$ is the set of all vertices of $X$ that span an edge with $v$. In particular, $v$ is not an element of its link. For any finite-dimensional simplex $\sigma$ let $\operatorname{Link}(\sigma)$ be the intersection of the links of each of the vertices in $\sigma$. We say that two vertices $\alpha, \beta$ in $\operatorname{Link}(\sigma)$ lie on the same side of $\sigma$ if there exists a vertex $\gamma$ in $\operatorname{Link}(\sigma)$ that fails to span an edge with both $\alpha$ and $\beta$, that is, if there exists a curve in $\operatorname{Link}(\sigma)$ that intersects both $\alpha$ and $\beta$. Observe that "being on the same 
side" defines an equivalence relation $\sim_{\sigma}$ on $\operatorname{Link}(\sigma)$, that is, the sides of $\sigma$ are the equivalence classes of $\sim_{\sigma}$ in $\operatorname{Link}(\sigma)$.

In particular, we may consider the sides of a vertex of $\mathcal{T}(\Sigma)$. We say that $\gamma$ in $\mathcal{T}(\Sigma)$ is $k$-sided if there are $k$ equivalence classes with respect to $\sim_{\gamma}$. As we shall see, $k$ is in $\{1,2\}$.

For any vertex $\gamma$ of $\mathcal{T}(\Sigma)$ there exist two subsurfaces $R, L \subset \Sigma$ obtained by cutting $\Sigma$ along $\gamma$ such that $\gamma$ is isotopic to the boundary components of both $R$ and $L$. Suppose $R$ is of finite type. We call $\gamma$ a pants curve if $\gamma$ is a separating curve and $R \cong \Sigma_{0,2}^{1}$, a sphere with two punctures and one boundary component. We call $\gamma$ a genus curve if $\gamma$ is a separating curve and $R \cong \Sigma_{1,0}^{1}$, a torus with one boundary component. If $\gamma$ is any other type of separating curve then we say it is type $X$.

If $\gamma$ is a bounding pair and one of the associated subsurfaces of $\Sigma$ is homeomorphic to $\Sigma_{1,0}^{2}$ then we call it a genus bounding pair.

Lemma 11. A vertex $\gamma$ in $\mathcal{T}(\Sigma)$ is 2-sided if and only if it is type $X$ or it is a genus bounding pair. Otherwise, $\gamma$ is 1-sided.

Proof. We first prove that if $\gamma$ has type $X$ then it has exactly two sides. Let $R$ and $L$ be the two subsurfaces of $\Sigma$ obtained by cutting along $\gamma$. Let $\alpha, \beta \in \operatorname{Link}(\gamma)$. If $\alpha \subset R$ and $\beta \subset L$, then any vertex of $\mathcal{T}(\Sigma)$ that intersects both $\alpha$ and $\beta$ must also intersect $\gamma$. This implies that $\gamma$ has at least two sides. If $\alpha, \beta \subset R$ then there exists an element of the $\operatorname{MCG}(\Sigma)$-orbit of $\alpha$ that intersects both $\alpha$ and $\beta$ and is contained in $R$. An identical argument holds for two vertices contained in $L$ and so it follows that $\gamma$ has exactly two sides.

Now let $\gamma$ be a genus one separating curve or a pants curve. Define $L, R \subset \Sigma$ as above. Observe that neither $\Sigma_{0,2}^{1}$ nor $\Sigma_{1,0}^{1}$ contains any nonperipheral separating curves or bounding pairs. Therefore Link $(\gamma)$ does not contain any curves in $R$. As above, all vertices contained in $L$ are on the same side and so $\gamma$ is 1 -sided.

We now move on to the case where $\gamma$ is a bounding pair. We define $R$ and $L$ as above. Assume that neither $R$ nor $L$ is homeomorphic to $\Sigma_{0,1}^{2}$ or $\Sigma_{1,0}^{2}$. Let $\alpha, \beta \in \operatorname{Link}(\gamma)$ be such that $\alpha \subset R$ and $\beta \subset L$. As shown in Figure 3, there exists a bounding pair $\gamma^{\prime}=\left\{\delta_{R}, \delta_{L}\right\}$ such that:

- any pair of curves in $\gamma$ or $\gamma^{\prime}$ forms a bounding pair,

- $\delta_{R} \subset R$ and $\delta_{L} \subset L$, and

- $\delta_{R} \cap \alpha \neq \emptyset$ and $\delta_{L} \cap \beta \neq \emptyset$.

That is, $\gamma^{\prime}$ is in $\operatorname{Link}(\gamma)$ and there is no edge between $\gamma^{\prime}$ and $\alpha$ or between $\gamma^{\prime}$ and $\beta$. It follows that $\gamma$ has exactly one side.

If $\gamma$ is a genus bounding pair then no such $\gamma^{\prime}$ exists. Indeed, every nonseparating curve in $R$ that forms a bounding pair with a curve in $\gamma$ is also isotopic to a curve in $\gamma$. By the same argument as for type $X$ vertices, we conclude that $\gamma$ is 2 -sided. 


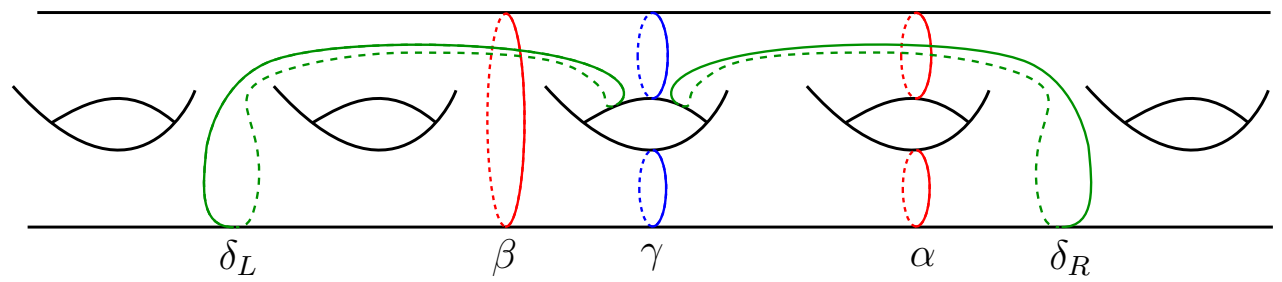

Figure 3. A general bounding pair $\gamma$ is 1-sided. For any two vertices $\alpha, \beta$ in $\mathcal{T}(\Sigma)$ adjacent to $\gamma$, we can find a bounding pair not adjacent to $\alpha$ and $\beta$ but adjacent to $\gamma$. Informally, bounding pairs can "pass through" each other.

If $R$ is homeomorphic to $\Sigma_{0,1}^{2}$ then $\gamma$ is 1 -sided. Indeed, the only vertex of $\mathcal{T}(\Sigma)$ contained in $R$ is $\gamma$ and so all vertices of $\operatorname{Link}(\gamma)$ are contained in $L$. This completes the proof.

Let $\sigma$ be a finite-dimensional simplex of $\mathcal{T}(\Sigma)$ consisting entirely of curves of type $X$. Using similar methods to the above proofs it is straightforward to show that the set of sides of $\sigma$ is in bijective correspondence with the subsurfaces of $\Sigma$ obtained by cutting $\Sigma$ along $\sigma$.

The following lemma follows immediately from Lemma 5 .

Lemma 12. Let $\sigma$ be a simplex in $\mathcal{T}(\Sigma)$. Then there are curves realizing the homotopy classes in $\sigma$ which do not accumulate in any compact set of $\Sigma$ if and only if for every vertex $v \notin \sigma$, the set $\{w \in \sigma \mid \mathrm{i}(v, w) \neq 0\}$ is finite.

Proof of Theorem 10. Let

$$
\Phi: \operatorname{MCG}(\Sigma) \rightarrow \operatorname{Aut}(\mathcal{T}(\Sigma))
$$

be the natural homomorphism; that is, for $f$ in $\operatorname{MCG}(\Sigma), \Phi(f)$ is the automorphism of $\mathcal{T}(\Sigma)$ determined by the rule

$$
\Phi(f)(\gamma)=f(\gamma)
$$

for every separating curve or bounding pair $\gamma$.

First, we show that $\Phi$ is injective. To this end, suppose $\Phi(f)=\mathrm{Id}$. Then we argue that $f(\gamma)=\gamma$ for every curve $\gamma$. Indeed, if $\gamma$ is separating, then $\gamma$ is a vertex of $\mathcal{T}(\Sigma)$, so $\Phi(f)(\gamma)=\gamma$ and we are done. If $\gamma$ is non-separating, there is some curve $\gamma^{\prime}$ such that $\gamma$ and $\gamma^{\prime}$ form a bounding pair. Because $\Phi(f)$ fixes the vertex corresponding to $\gamma \cup \gamma^{\prime}$, it must be the case that either $f(\gamma)=\gamma$ and $f\left(\gamma^{\prime}\right)=\gamma^{\prime}$ or $f(\gamma)=\gamma^{\prime}$ and $f\left(\gamma^{\prime}\right)=\gamma$. But there exists a separating curve $\eta$ that intersects $\gamma$ but not $\gamma^{\prime}$. Because $f(\eta)=\eta$, it cannot be the case that $f(\gamma)=\gamma^{\prime}$. Therefore $f(\gamma)=\gamma$ as desired.

By the Alexander method for infinite-type surfaces, due to HernándezMoralez-Valdez [13], we deduce that $f$ is the identity in $\operatorname{MCG}(\Sigma)$.

We now show that $\Phi$ is surjective. Let $\phi: \mathcal{T}(\Sigma) \rightarrow \mathcal{T}(\Sigma)$ be an automorphism. Fix a principal exhaustion $\left\{P_{1}, P_{2}, \ldots\right\}$ of $\Sigma$ such that $P_{1}$ has 
complexity at least six. Define $\sigma_{i}$ to be the simplex of $\mathcal{T}(\Sigma)$ corresponding to the multicurve $\partial P_{i}$. Denote by $\mathcal{P}_{i}$ the subcomplex of $\mathcal{T}(\Sigma)$ spanned by the curves and bounding pairs contained in $P_{i}$. Denote by $P_{i}^{\circ}$ the surface obtained by gluing once-punctured disks to each boundary component of $P_{i}$. By construction, $\sigma_{i}$ contains only type $X$ vertices and therefore $\mathcal{T}\left(\mathcal{P}_{i}\right)$ is isomorphic to $\mathcal{T}\left(P_{i}^{\circ}\right)$. As the complexity of $P_{i}$ is at least six, $\mathcal{P}_{i}$ is connected for all $i$. By Lemma 9, we know that $\mathcal{P}_{i}$ is the unique side of $\sigma_{i}$ whose diameter is infinite.

Since $\phi$ is a simplicial automorphism, it induces a bijection between the sides of $\sigma_{i}$ and the sides of $\phi\left(\sigma_{i}\right)$. Because all simplicial automorphisms of $\mathcal{T}(\Sigma)$ are isometries, $\phi\left(\sigma_{i}\right)$ has a unique side of infinite diameter. From Lemma 11 we have that every vertex of $\phi\left(\sigma_{i}\right)$ is of type $X$ or it is a genus bounding pair. However, since each side of $\phi\left(\sigma_{i}\right)$ is connected, it cannot contain a genus bounding pair by Lemma 11 .

We write $\mathcal{Q}_{i} \subset \operatorname{Link}\left(\phi\left(\sigma_{i}\right)\right)$ for the unique side of $\phi\left(\sigma_{i}\right)$ with infinite diameter, and $Q_{i} \subset \Sigma$ for the finite-type subsurface which it defines. By Lemma 12 , we can realize the curves $\bigcup \mathcal{Q}_{i}$ by non-accumulating curves in $\Sigma$. The $Q_{i}$ 's form a sequence of nested subsurfaces, thus their union is an open set. The nonaccumulation property implies that $\bigcup Q_{i}$ is the full surface $\Sigma$.

Since each vertex of $\phi\left(\sigma_{i}\right)$ has type $X$ we have that $\mathcal{Q}_{i} \cong \mathcal{T}\left(Q_{i}^{\circ}\right)$. Furthermore, $\phi$ restricts to an isomorphism

$$
\phi_{i}: \mathcal{P}_{i} \rightarrow \mathcal{Q}_{i} .
$$

Since each $P_{i}$ is assumed to have complexity at least six, the combination of results of Kida [16] and Korkmaz [18] implies that $\phi_{i}$ is induced by a homeomorphism $f_{i}: P_{i} \rightarrow Q_{i}$. Moreover, the homeomorphism $f_{i+1}$ may be chosen so that it restricts to $f_{i}$ on the subsurface $P_{i}$. Hence their direct limit is a homeomorphism of $\bigcup P_{i}$ to $\bigcup Q_{i}$ inducing $\phi$. Since $\Sigma=\bigcup P_{i}=\bigcup Q_{i}$, this completes the proof.

We now end this subsection with the following two observations which will be useful later.

Lemma 13. For any simplex $\sigma$ in $\mathcal{T}(\Sigma)$ and any compactly-supported $f \in$ $\mathcal{I}(\Sigma)$, if $f$ preserves $\sigma$ then $f$ fixes $\sigma$ pointwise.

Proof. Consider two vertices $v, w \in \sigma$, and assume $f(v)=w$. Then $v$ and $w$ are either both separating curves or they are both bounding pairs.

Suppose $v$ separates $\Sigma$ into two infinite-type subsurfaces. Since $v$ and $w$ are disjoint, we can find a compact domain $Y \subset \Sigma$ such that $v$ and $w$ are of different topological type. By choosing $Y$ large enough, we can assume that $Y$ supports $f$. But then it is impossible for $\left.f\right|_{Y}(v)=f(v)=w$.

Suppose now that $v$ (and $w$ ) separates $\Sigma$ into a finite-type subsurface and an infinite-type subsurface. Call the finite-type subsurfaces $V$ and $W$, corresponding to $v$ and $w$ respectively. Since $v \in \Sigma \backslash W$ and $w \in \Sigma \backslash V$, the subsurfaces $V$ and $W$ are disjoint. By assumption $f(v)=w$, therefore 
$V$ and $W$ must have the same topological type and $f(V) \subset W$. But this is impossible if $f \in \mathcal{I}(\Sigma)$.

Lemma 14. For any bounding pair $v \in \mathcal{T}(\Sigma)$ and any $f \in \mathcal{I}(\Sigma)$, if $f$ preserves $v$, then $f$ must fix the curves in $v$.

Proof. Suppose $v=\{\alpha, \beta\}$ and $f(\alpha)=\beta$. The complement of $v$ in $\Sigma$ has two components. Since $f$ is pure it preserves the components. Let $Y$ be one of the components. The orientation of $Y$ induces an orientation on $\alpha$ and $\beta$. These oriented curves (as homology classes) satisfy $\alpha=-\beta$. The map $f$ is orientation preserving, so it must preserve the orientation of $\alpha$ and $\beta$. That is, $f(\alpha)=\beta$ as oriented curves. But then $\alpha$ and $f(\alpha)$ cannot be homologous, contradicting that $f \in \mathcal{I}(\Sigma)$.

4.3. Algebraic characterization of twists and bounding pair maps. Before proving Theorem 4 we will need one more ingredient. Notice that the vertices of $\mathcal{T}(\Sigma)$ define supports of elements in $\mathcal{I}(\Sigma)$. We must now show that commensurations of $\mathcal{I}(\Sigma)$ preserve such elements and therefore define a permutation of the vertices of the complex. We will adapt the algebraic characterization of Dehn twists of Bavard-Dowdall-Rafi [2] to our setting.

We first introduce some terminology to facilitate the characterization of twists and bounding pairs. Let $G<\operatorname{MCG}(\Sigma)$. We denote by $\mathcal{F}_{G}$ the set of elements of $G$ whose conjugacy class (in $G$ ) is countable. Bavard-DowdallRafi prove that if $G$ is finite-index in $\operatorname{MCG}(\Sigma)$ then $f$ is in $\mathcal{F}_{G}$ if and only if it has compact support [2, Proposition 4.2]. Using similar methods, we will show:

Proposition 15. Let $G<\mathcal{I}(\Sigma)$ be a finite-index subgroup. An element $f$ in $G$ has compact support if and only if $f$ is in $\mathcal{F}_{G}$.

Proof. It is clear that compactly-supported mapping classes have countable conjugacy classes. For the opposite direction, the argument in [2, Proposition 4.2] exhibits a infinite sequence of pairwise-disjoint curves $a_{i}$ such that the Dehn twists about the $a_{i}$ give rise to uncountably many conjugates of $f$. Since $\Sigma$ has infinite type, the curves $a_{i}$ may be chosen to be separating, so that the corresponding twists belong to $\mathcal{I}(\Sigma)$. Hence the result follows.

We denote by $Z(H)$ the center of $H$. If $h$ is in $H$, we write $C_{H}(h)$ for the centralizer of $h$ in $H$. Given a finite-index subgroup $G<\mathcal{I}(\Sigma)$ we write $\mathcal{M}_{G}$ for the set of elements $f$ in $G$ which satisfy the following three conditions:

(1) $f \in \mathcal{F}_{G}$,

(2) $Z\left(\mathcal{F}_{G} \cap C_{G}(f)\right)$ is an infinite cyclic group, and

(3) $C_{G}(f)=C_{G}\left(f^{k}\right)$ for every $k>0$.

We now prove that, for any finite-index subgroup $G$ of $\mathcal{I}(\Sigma)$, powers of Dehn twists and bounding pair maps belong to the set $\mathcal{M}_{G}$.

Lemma 16. Let $G<\mathcal{I}(\Sigma)$ be a finite-index subgroup. If $f \in G$ is a power of a Dehn twist about a separating curve or a bounding pair map then $f$ belongs to $\mathcal{M}_{G}$. 
Proof. Since $f$ has compact support, $f \in \mathcal{F}_{G}$. Suppose first $f$ is a power of a Dehn twist about the separating curve $\gamma$. We have that

$$
C_{\mathcal{I}(\Sigma)}\left(T_{\gamma}^{k}\right)=\{g \in \mathcal{I}(\Sigma) \mid g(\gamma)=\gamma\}
$$

for $k \neq 0$. It follows that all powers of $T_{\gamma}$ have the same centralizer in $\mathcal{I}(\Sigma)$ and hence, in any subgroup. A similar argument holds if $f$ is a power of bounding pair map. This implies the third condition in the definition of $\mathcal{M}_{G}$.

To see that $f$ satisfies the second condition, once again assume first that $f$ is a power of the Dehn twist about a separating curve $\gamma$. Let $g$ be a nontrivial element of $\mathcal{F}_{G} \cap C_{G}(f)$ and assume that $g$ is not a power of $T_{\gamma}$. Then there exists a curve $\delta$ disjoint from $\gamma$ such that $g(\delta) \neq \delta$. If $\delta$ is a separating curve then $T_{\delta}^{k}$ is in $C_{G}(f)$, for some $k>0$, but $g T_{\delta}^{k} \neq T_{\delta}^{k} g$, so $g$ is not in $Z\left(\mathcal{F}_{G} \cap C_{G}(f)\right)$. On the other hand, suppose $\delta$ is a nonseparating curve; since $g$ acts trivially on homology we have that $\delta$ and $g(\delta)$ are homologous curves. Because $T_{\gamma} g(\delta)=g T_{\gamma}(\delta)=g(\delta)$, we have that $g(\delta)$ is disjoint from $\gamma$. It follows that some power of $T_{\delta} T_{g(\delta)}^{-1}$ belongs to $C_{G}(f)$, but does not commute with $g$. We have therefore shown that $g$ is not central in $\mathcal{F}_{G} \cap C_{G}(f)$ as desired.

A similar argument also shows that, if $f$ is a power of a bounding pair map, then $g$ belongs to $Z\left(\mathcal{F}_{G} \cap C_{G}(f)\right)$ if and only if it is a power of the same bounding pair map.

When $G$ is a finite-index subgroup of $\operatorname{MCG}(\Sigma)$, all elements of $\mathcal{M}_{G}$ are powers of multi Dehn twists, see [2, Lemma 4.5]. In stark contrast, this is no longer true in our setting. When $G$ is a finite-index subgroup of $\mathcal{I}(\Sigma)$, then $\mathcal{M}_{G}$ may contain elements which are not supported on a disjoint union of annuli: for example, we may take a pure braid on a nonseparating planar subsurface with at least three boundary components. The following proposition characterizes those elements that lie in $\mathcal{M}_{G}$, when $G$ is a finiteindex subgroup of $\mathcal{I}(\Sigma)$. Recall the definition of $\partial f$ for a mapping class $f$, which is non-empty when $f$ is compactly-supported. We have the following statements.

Lemma 17. Let $G<\mathcal{I}(\Sigma)$ be a finite-index subgroup. Given a nontrival $f \in \mathcal{M}_{G}$, let $\sigma \subset \partial f$ be the set of all separating curves or bounding pairs. The following statements hold.

(1) For all $g \in \mathcal{F}_{G} \cap C_{G}(f)$, g fixes every component of $\Sigma \backslash \partial f$.

(2) If $\sigma$ is empty, then for each finite-type domain $Y$ of $\Sigma \backslash \partial f,\left.f\right|_{Y}$ is either the identity map or pseudo-Anosov. Furthermore, there is at least one pseudo-Anosov component of $f$.

(3) If $\sigma$ is non-empty then $\sigma=\partial f$ is either a separating or a bounding pair, and $f=T_{\sigma}^{j}$ for some non-zero $j$, where $T_{\sigma}$ is either a separating twist or a bounding pair map. 
Proof. For any $g \in \mathcal{F}_{G} \cap C_{G}(f), g(\partial f)=\partial\left(g f g^{-1}\right)=\partial f$. To show that $g$ fixes every component of $\Sigma \backslash \partial f$, it is enough to show $g$ fixes each component of $\partial f$. To see this, suppose there exists a domain $Y$ of $\Sigma \backslash \partial f$ such that $g(Y)$ is not $Y$. Since $g$ fixes the components of $\partial f, Y$ and $g(Y)$ share the same boundary. This means $\Sigma=Y \cup g(Y) \cup \partial Y$, so $Y$ must have infinite type. But then $g$ cannot be a pure mapping class, contradicting the fact that $g \in \mathcal{I}(\Sigma)$. In the following, we will consider the two cases that $\sigma$ is empty or $\sigma$ is not empty. For each case, we show $g$ fixes the components of $\partial f$ along with the statements of (2) and (3).

First suppose that $\sigma$ is empty. In this case, every curve of $\partial f$ is nonseparating and no two form a bounding pair. In particular, no two curves of $\partial f$ are homologous, so every element of $\mathcal{F}_{G} \cap C_{G}(f)$ must fix the components of $\partial f$. Now let $Y$ be a component of $\Sigma \backslash \partial f$ of finite-type. The map $\left.f\right|_{Y}$ is irreducible (otherwise the reducing curves would also be in $\partial f$ ), so it is either of finite order or pseudo-Anosov. We now show it is not possible for $\left.f\right|_{Y}$ to be non-trivial and have finite order. Since $\partial f$ does not contain any separating curves or bounding pairs, it has cardinality at least 3 . This means $\chi(Y)<0$, in which case $\mathrm{MCG}(Y)$ is torsion-free ([10, Corollary 7.3]). Hence, if $\left.f\right|_{Y}$ is not pseudo-Anosov, then $\left.f\right|_{Y}$ is identity. Finally, if there is no pseudo-Anosov component of the support of $f$, then there exists a power $k \geq 1$ such that $\left.f^{k}\right|_{Y}$ is identity for any component $Y$ in $\Sigma \backslash \partial f$. But $f$ has infinite order by Lemma 6 , so $f^{k}$ must be a product of powers of Dehn twists about curves in $\partial f$. This is impossible as $f \in \mathcal{I}(\Sigma)$ [28].

Now suppose $\sigma$ is non-empty. Regard $\sigma$ as a simplex in $\mathcal{T}(\Sigma)$. Every element $g \in \mathcal{F}_{G} \cap C_{G}(f)$ preserves $\partial f$, and hence also $\sigma$. By Lemma $13, g$ fixes each vertex of $\sigma$. Then, by Lemma 14, we can further conclude that $g$ also fixes each curve in $\sigma$. Let $v$ by a vertex of $\sigma$, and let $T_{v}$ be the twist about $v$, which belongs to $\mathcal{I}(\Sigma)$.

We now show $f=T_{v}^{j}$ for some non-zero $j$. In particular, $v=\sigma=\partial f$. Since every $g \in \mathcal{F}_{G} \cap C_{G}(f)$ preserves $v, g$ commutes with $T_{v}$, showing $T_{v}^{k} \in Z\left(\mathcal{F}_{G} \cap C_{G}(f)\right)$ for some $k \geq 1$. Since $f$ also lies in $Z\left(\mathcal{F}_{G} \cap C_{G}(f)\right)$ which is infinite cyclic by assumption, we must have $f^{m}=T_{v}^{n}$ for some nonzero integers $m$ and $n$. Let $w$ a vertex of $\mathcal{T}(\Sigma)$ disjoint from $v$ and choose $k \geq 1$ so that $T_{w}^{k} \in G$. Since $v$ and $w$ are disjoint, we have

$$
T_{w}^{k} \in C_{G}\left(T_{v}^{n}\right)=C_{G}\left(f^{m}\right)=C_{G}(f) .
$$

This is only possible if $f(w)=w$. Thus, $f$ fixes every separating curve and bounding pair disjoint from $v$. Applying Lemma 14, we obtain that $f$ fixes every curve disjoint from $v$. This shows $f=T_{v}^{j}$ for some non-zero integer $j$, concluding the proof.

Definition (Essential Support). For each element $f \in \mathcal{M}_{G}$, we define the essential support $\operatorname{supp}(f)$ of $f$ as follows. Let $\sigma \subset \partial f$ be the set of all separating curves or bounding pairs.

- If $\sigma$ is nonempty, then $\operatorname{supp}(f)=\sigma$. 
- If $\sigma$ is empty, then $\operatorname{supp}(f)$ is the union over all domains $Y$ in $\Sigma \backslash \partial(f)$ such that $\left.f\right|_{Y}$ is a pseudo-Anosov. Note that by Lemma $17, \operatorname{supp}(f)$ is nonempty.

Let

$$
C_{\mathcal{M}_{G}}(f)=\left\{g \in \mathcal{M}_{G} \mid f g=g f\right\} .
$$

We also define a further subset:

$$
\left(\mathcal{P}_{G}\right)_{f}=\left\{g \in C_{\mathcal{M}_{G}}(f) \mid g \text { is supported in } \Sigma \backslash \operatorname{supp}(f)\right\} .
$$

The next lemma tells us that the elements supported in $\operatorname{supp}(f)$ are precisely those that are central.

Lemma 18. Let $G<\mathcal{I}(\Sigma)$ be a finite-index subgroup. For any element $f$ in $\mathcal{M}_{G}$ we have that

$$
C_{\mathcal{M}_{G}}(f)=Z\left(C_{\mathcal{M}_{G}}(f)\right) \oplus\left(\mathcal{P}_{G}\right)_{f}
$$

Proof. Let $\sigma \subset \partial f$ be the set of all separating curves or bounding pairs. If $\sigma$ is nonempty, then by Lemma $17, \sigma=\partial f$ is a separating curve or a bounding pair, and $f=T_{\sigma}^{j}$, where $T_{\sigma}$ is a separating twist or a bounding pair map. In this case, any element $g \in C_{\mathcal{M}_{G}}(f)$ fixes every curve of $\sigma$. Thus, if $g$ has support in $\operatorname{supp}(f)$, then $g$ is itself a power of $T_{\sigma}$. This shows $g \in Z\left(C_{\mathcal{M}_{G}}(f)\right)$.

Now suppose $\sigma$ is empty. By Lemma $17, \operatorname{supp}(f)$ is non-empty. Let $g \in C_{\mathcal{M}_{G}}(f) \subset \mathcal{F} \cap C_{G}(f)$ be an element with support in $\operatorname{supp}(f)$. We want to show $g$ lies in the center of $C_{\mathcal{M}_{G}}(f)$. If $h \in C_{\mathcal{M}_{G}}(f)$ has support disjoint from $\operatorname{supp}(f)$, then $h$ and $g$ clearly commute. Henceforth, we may assume this is not the case.

By Lemma 17, $f, g$, and $h$ all preserve every component of $\operatorname{supp}(f)$. Thus, we can assume without a loss of generality that $h$ is also supported in $\operatorname{supp}(f)$. Let $Y_{1}, \ldots, Y_{n}$ be the components of $\operatorname{supp}(f)$. For each $i$, let $f_{i}$, (resp. $g_{i}$ and $h_{i}$ ) denote the restriction of $f$ (resp. $g$ and $h$ ) to $Y_{i}$. Since the components of $\partial f$ are non-separating and no two form a bounding pair, we can write

$$
f=f_{1} f_{2} \cdots f_{n} \quad g=g_{1} g_{2} \cdots g_{n}, \quad h=h_{1} h_{2} \cdots h_{n},
$$

where each $f_{i}$ is pseudo-Anosov on $Y_{i}$. Since $\operatorname{MCG}\left(Y_{i}\right)$ is torsion-free and $f \in \mathcal{M}_{G}$, the centralizer of $f_{i}$ in $\operatorname{MCG}\left(Y_{i}\right)$ is cyclic. As $f$ commutes with $g$ and $h$, each $f_{i}$ commutes with $g_{i}$ and $h_{i}$. Thus, each $g_{i}$ or $h_{i}$ is contained in the centralizer of $f_{i}$, yielding $g_{i}$ and $h_{i}$ commute for all $i$. This shows $g$ and $h$ commute as required.

Finally, we can prove the characterization of Dehn twists and bounding pair maps.

Proposition 19. Let $G<\mathcal{I}(\Sigma)$ be a finite-index subgroup, and let $f$ lie in $G$. Then $f$ is a power of a Dehn twist or of a bounding pair map if and only $f$ is in $\mathcal{M}_{G}$, and for all $g$ in $\mathcal{M}_{G}$ such that $\left(\mathcal{P}_{G}\right)_{g}=\left(\mathcal{P}_{G}\right)_{f}$ we have that there exist integers $i, j \neq 0$ such that $f^{i}=g^{j}$. 
Proof. The forward direction is Lemma 16 and the definition of $\left(\mathcal{P}_{G}\right)_{f}$.

For the other direction, we prove the contrapositive. Assume that $f$ is not a power of a Dehn twist or bounding pair map. Our goal is to find an element $g \in \mathcal{M}_{G}$ with $\operatorname{supp}(g)=\operatorname{supp}(f)$ such that no powers of $f$ and $g$ are equal, but $\left(\mathcal{P}_{G}\right)_{f}=\left(\mathcal{P}_{G}\right)_{g}$.

By Lemma 17, there exists a domain $Y$ in $\Sigma \backslash \partial f$ on which $\left.f\right|_{Y}$ is a pseudo-Anosov. Since $Y$ supports a pseudo-Anosov, we may choose an element $h \in \operatorname{MCG}(\Sigma)$ such that $h$ preserves the components of $\Sigma \backslash \partial f$, is identity outside of $Y$, and the restriction to $Y$ of $f$ and $g=h f h^{-1}$ are two independent pseudo-Anosovs. Since $\mathcal{I}(\Sigma)$ is normal in $\operatorname{MCG}(\Sigma), g \in \mathcal{I}(\Sigma)$, and by construction, $\operatorname{supp}(g)=\operatorname{supp}(f)$. Thus $\left(\mathcal{P}_{G}\right)_{g}=\left(\mathcal{P}_{G}\right)_{f}$, but $f$ and $g$ do not have a common power.

4.4. Abstract commensurators of the Torelli group. We can now finally prove Theorem 4 . For a bounding pair $\gamma=\left\{\gamma_{1}, \gamma_{2}\right\}$ we use the shorthand $T_{\gamma}$ for the bounding pair map $T_{\gamma_{1}} T_{\gamma_{2}}^{-1}$.

Proof of Theorem 4. Let $[\psi]$ be an element of $\operatorname{Comm} \mathcal{I}(\Sigma)$ representing the isomorphism of finite index subgroups

$$
\psi: G_{1} \rightarrow G_{2} .
$$

Let $\gamma$ be a separating curve or a bounding pair and choose $n$ in $\mathbb{N}$ so that $T_{\gamma}^{n}$ is in $G_{1}$. By Proposition 19, $T_{\gamma}^{n}$ is in $\mathcal{M}_{G_{1}}$ and for all $g$ in $\mathcal{M}_{G_{1}}$ such that $\left(\mathcal{P}_{G_{1}}\right)_{g}=\left(\mathcal{P}_{G_{1}}\right)_{T_{\gamma}^{n}}$, there exist integers $i, j$ so that $\left(T_{\gamma}^{n}\right)^{i}=g^{j}$. Since these conditions are preserved by isomorphism, we have that $\psi\left(T_{\gamma}^{n}\right)$ lies in $\mathcal{M}_{G_{2}}$, Proposition 19 implies there exists a separating curve or bounding pair $\delta$ and a nonzero integer $m$ such that $\psi\left(T_{\gamma}^{n}\right)=T_{\delta}^{m}$.

At this point, and again with respect to the above notation, we obtain that $\psi$ induces a map

$$
\begin{aligned}
\psi_{*}: \mathcal{T}(\Sigma) & \rightarrow \mathcal{T}(\Sigma) ; \\
\gamma & \mapsto \delta .
\end{aligned}
$$

We observe that $\psi_{*}$ is a simplicial map, since powers of Dehn twists and bounding pair maps commute if and only if the underlying curves are disjoint. Moreover, the map is also bijective, with inverse the simplicial map associated to the inverse of $\psi^{-1}$.

By Theorem 10, there exists an $f \in \operatorname{MCG}(\Sigma)$ such that $\psi_{*}(\gamma)=f(\gamma)$ for every separating curve or bounding pair $\gamma$. Now, for any $g$ in $G_{1}$ we have

$$
\psi\left(g T_{\gamma}^{n} g^{-1}\right)=\psi(g) \psi\left(T_{\gamma}^{n}\right) \psi\left(g^{-1}\right)=\psi(g) T_{f(\gamma)}^{n} \psi\left(g^{-1}\right)=T_{\psi(g) f(\gamma)}^{n},
$$

and therefore

$$
T_{\psi(g) f(\gamma)}^{n}=\psi\left(g T_{\gamma}^{n} g^{-1}\right)=\psi\left(T_{g(\gamma)}^{n}\right)=T_{f g(\gamma)}^{n} .
$$

Therefore $\psi(g) f(\gamma)=f g(\gamma)$. By use of the Alexander method [13] we conclude that $\psi(g)=f g f^{-1}$. This shows that every abstract commensurator 
of $\mathcal{I}(\Sigma)$ is defined by conjugation by a mapping class of $\Sigma$, and in particular, so is every automorphism of $\mathcal{I}(\Sigma)$.

On the other hand, suppose there exists an $f$ in $\operatorname{MCG}(\Sigma)$ and a finiteindex subgroup $H<\mathcal{I}(\Sigma)$ such that conjugation by $f$ induces the identity map on $H$. For any separating curve or bounding pair $\gamma$, there exists some $m \geq 1$ such that $T_{\gamma}^{m}$ lies in $H$. Thus

$$
T_{\gamma}^{m}=f T_{\gamma}^{m} f^{-1}=T_{f \gamma}^{m} .
$$

By [2, Lemma 2.5], $f \gamma=\gamma$, and thus $f$ is the identity by Theorem 10 . This completes the proof.

\section{REFERENCES}

[1] J. Aramayona, P. Patel, and N. G. Vlamis. The first integral cohomology of pure mapping class groups. ArXiv e-prints, November 2017.

[2] Juliette Bavard, Spencer Dowdall, and Kasra Rafi. Isomorphisms between big mapping class groups. I.M.R.N., in press.

[3] Mladen Bestvina, Kai-Uwe Bux, and Dan Margalit. The dimension of the Torelli group. J. Amer. Math. Soc., 23(1):61-105, 2010.

[4] Joan S. Birman. On Siegel's modular group. Math. Ann., 191:59-68, 1971.

[5] Tara Brendle and Dan Margalit. Normal subgroups of mapping class groups and the metaconjecture of ivanov.

[6] Tara E. Brendle and Dan Margalit. Commensurations of the Johnson kernel. Geometry and Topology, (8):1361-1384, 2004.

[7] Tara E. Brendle and Dan Margalit. Addendum to: "Commensurations of the Johnson kernel" [Geom. Topol. 8 (2004), 1361-1384; mr2119299]. Geom. Topol., 12(1):97-101, 2008.

[8] Heinrich Burkhardt. Grundzüge einer allgemeinen Systematik der hyperelliptischen Functionen I. Ordnung. Math. Ann., 35(1-2):198-296, 1889.

[9] Benson Farb and Nikolai V. Ivanov. The Torelli geometry and its applications. research announcement. Mathematical Research Letters, 293-301, 2005.

[10] Benson Farb and Dan Margalit. A primer on mapping class groups. Princeton University Press, 2012.

[11] Michael Handel and William P. Thurston. New proofs of some results of Nielsen. Adv. in Math., 56(2):173-191, 1985.

[12] Allen Hatcher and Dan Margalit. Generating the Torelli group. Enseign. Math. (2), 58(1-2):165-188, 2012.

[13] Jesus Hernández Hernández, Isreal Morales, and Ferrán Valdez. The Alexander method for infinite-type surfaces. arXiv:1703.0040\%, 2017.

[14] Nikolai V. Ivanov. Automorphisms of complexes of curves and of Teichmüller spaces. International Mathematics Research Notices, (14):651-666, 1997.

[15] Dennis L. Johnson. Homeomorphisms of a surface which act trivially on homology. Proc. Amer. Math. Soc., 75(1):119-125, 1979.

[16] Yoshikata Kida. Automorphisms of the Torelli complex and the complex of separating curves. Journal of the Mathematical Society of Japan, 63, 2011.

[17] Yoshikata Kida. The co-Hopfian property of the Johnson kernel and the Torelli group. Osaka J. Math., 50(2):309-337, 2013.

[18] Mustafa Korkmaz. Automorphisms of complexes of curves on punctured spheres and on punctured tori. Topology and its applications, 95, 1999.

[19] Feng Luo. Automorphisms of the complex of curves. Topology, 39, 2000.

[20] Darryl McCullough and Andy Miller. The genus 2 Torelli group is not finitely generated. Topology Appl., 22(1):43-49, 1986. 
[21] Alan McLeay. Geometric normal subgroups in mapping class groups of punctured surfaces.

[22] Geoffrey Mess. The Torelli groups for genus 2 and 3 surfaces. Topology, 31(4):775-790, 1992.

[23] Priyam Patel and Nicholas G. Vlamis. Algebraic and topological properties of big mapping class groups. Algebraic and Geometric Topology, in press.

[24] Jerome Powell. Two theorems on the mapping class group of a surface. Proc. Amer. Math. Soc., 68(3):347-350, 1978.

[25] Andrew Putman. Cutting and pasting in the torelli group. Geom. Topol., 11(2):829$865,2007$.

[26] Andrew Putman. Small generating sets for the Torelli group. Geom. Topol., 16(1):111-125, 2012.

[27] Ian Richards. On the classification of noncompact surfaces. Trans. Amer. Math. Soc., 106:259-269, 1963.

[28] William R. Vautaw. Abelian subgroups of the Torelli group. Algebr. Geom. Topol., 2:157-170, 2002.

Universidad Autónoma de MAdrid \& ICMAT, MAdrid, Spain

E-mail address: aramayona@gmail.com

Department of Mathematics, University of Manitoba, Winnipeg, Canada

E-mail address: ty.ghaswala@gmail.com

Department of Mathematics, University of Wisconsin, Madison, Wi, United STATES

E-mail address: kent@math.wisc.edu

Mathematics Research Unit, University of Luxembourg, Esch-Sur-Alzette, LUXEMBOURG

E-mail address: mcleay.math@gmail.com

Department of Mathematics, University of Oklahoma, Norman, OK, United STATES

E-mail address: jing@ou.edu

Department of Mathematics, University of Michigan, Ann Arbor, Mi, United STATES

E-mail address: rebecca.winarski@gmail.com 